\title{
sciendo
}

\author{
MARIA PYDA-KARWICKA ${ }^{1,2}$, MALWINA KARWICKA ${ }^{1}$, MAGDALENA ORŁOWSKA ${ }^{3}$, \\ MARIUSZ KRATA ${ }^{4}$, AGNIESZKA PEDRYCZ ${ }^{5}$
}

\section{Renal-cell carcinoma - novel concepts of classification}

\begin{abstract}
Renal cancer, according to the Polish National Cancer Registry, was the cause of $4 \%$ of cancer cases in men and 3\% of cases in women in 2010. Most often it affects men over 65, although it may also concern infants. In 2010, the number of reported cases for men was 2,700 and 1,900 for women. For the last three decades - up to 1990, the incidence of kidney cancer has increased significantly. Currently, it remains at the stable level of morbidity. One of the most significant issues of modern diagnostics and treatment of renal cancer is the problem of its heterogeneity brin-ging many difficulties in systematization and categorization of this neoplasm. The World Health Organization Classification of Renal Tumors has been published in 2004 and is currently supplemented with new classification units, which, according to ISUP (International Society of Urological Pathology), should be considered as separate groups of kidney epithelial cancers.
\end{abstract}

Keywords: renal cell carcinoma, novel cancer classification, PRCC characteristic.

DOI: $10.2478 /$ pjph-2019-0014

\section{INTRODUCTION}

This paper contains an outline of the most important aspects related to the diagnosis of renal cancer. The work is a review paper, based on Polish and English literature as well as the analysis of reports prepared by the International Society of Urological Pathology (ISUP) Consensus Conference on Renal Tumors, which took place in 2012 in Vancouver, Canada.

In our paper we discussed the most important issues regarding conventional diagnostic methods, as well as a few modern diagnostic approaches with a potential in clinical practice.

\section{Anatomy and histology}

The kidney is an organ with an average weight of $110-170 \mathrm{~g}$ and average dimensions: $12 \times 6 \times 4-5 \mathrm{~cm}$, in proportion to body weight and sex, lying on the right and left side, on the back wall of the abdominal cavity, right below the diaphragm.

It is covered with a fibrous capsule and layers of surrounding fat tissue, forming together the so-called Greta's fascia, protecting the kidney from shocks and injuries. The shape of the kidney resembles bean seed with its convex part directed to the side and the hilum located on the concave border, containing the ureters, blood and lymph vessels and hilar lymph nodes.

On the cross-section one can distinguish the external cortical part and the medulla, made of renal pyramids whose bases define the border between the cortex and the medulla. Each pyramid, along with the surrounding fragment of the cortex, forms a structural unit called the renal lobe.

The basic functional unit of kidney is the nephron, composed of renal corpuscle and system of cortical and medullary tubules transporting the glomerular filtrate to the renal pelvis. The tubular and collecting system of the nephron is consisted of proximal and distal, convoluted and straight sections. Histologically, the basic structure of the convoluted tubule wall is similar along the entire length of the tubule - it consists of tightly packaged cuboidal epithelial cells on the basement membrane, although epithelial cells lining the inner parts of the tubule have various functions in specific tubule parts.

A similar construction also applies to straight section called Henle's loop, however, the epithelium lining the ascending as well as descending part is a single-layered epithelium.

\section{Renal neoplasms}

Renal cancer, according to the Polish National Cances Registry, was the cause of $4 \%$ of cancer cases in men and $3 \%$ of cases in women in 2010 [1]. As many as $90 \%$ of kidney malignant neoplasms are cancers derived from the renal tubular epithelium. As regards the most frequent histological type of renal cell carcinoma, it is clear cell renal cell carcinoma derived from the epithelial cells of the proximal tubule of the nephron [2]. In macroscopic observations, it is a clearly delineated tumor often yellow colored, resembling the adrenal gland. Hemorrhages are often present, and sometimes extensive necrosis.

\footnotetext{
${ }^{1}$ School of Medicine Jan Kochanowski University in Kielce, Poland

${ }^{2}$ Holy Cross Cancer Center Kielce, Poland

${ }^{3}$ International Institute of Molecular and Cell Biology in Warsaw, Poland

${ }^{4}$ Internal Medicine Ward, Independent Public Healthcare Centre Szczebrzeszyn, Poland

${ }^{5}$ Chair and Department of Histology and Embryology with Experimental Cytology Unit, Medical University in Lublin, Poland
} 
The basic morphological feature that is used in diagnosis of clear cell carcinoma is the characteristic abundant, light or slightly acidophilic cytoplasm of cancer cells. Cancer cells form lobular or vesicular patterns, surrounded by a network of tiny, thin-walled blood vessels. They often have a centrally located round cell nucleus containing the nucleolus. The size of the nucleus and the appearance of nucleoli are included in the classification of cancer in the four-level scale of the so-called cancer grading according to Dr. Joel Fuhrman [3].

The occurrence of necrosis within the tumor and the process of sarcomatoid dedifferentiation, which means a presence of solid tumor fields with specific growth pattern characterized by spindle-shaped cell morphology with features of increased atypia and mitoses, are also prognostically unfavorable factors. Papillary renal cell carcinoma is the second in terms of incidence renal cancer.

As in the case of clear cell carcinoma, its cells are phenotypically and genotypically differentiated towards the epithelial cells of the proximal tubule of the nephron. It can be multifocal and occur in both kidneys. Usually the tumor is well demarcated, in larger ones blood hemorrhages and areas of necrosis can be observed. The basic feature of the microscopic image of the tumor is a presence of papillary structures. However, the histological structure of this type of neoplasm is often heterogeneous. Delahunt et al. distinguished two types of papillary renal cell carcinoma, considering the size of tumor cells, the content and nature of the cytoplasm, and the microscopic pattern of the tumor [4].

In Type I, the tumor cells are small, with a small amount of mostly basophilic cytoplasm, forming a cellular monolayer. In type II papillary renal cell carcinoma, tumor cells contain abundant, acidophilic cytoplasm and often form pseudomultilayered systems; their cell nuclei are usually larger, with a clearly marked polymorphism. Moreover, there are papillary renal cell carcinomas, primarily eosinophilic, which cannot be classified into any of these types. However, there is no consensus for the formation of the third type of papillary carcinoma for these cancers [5].

Malignant renal neoplasm with differentiation towards the epithelial cells of the distal nephron tubule is chromophobe cell renal carcinoma. It refers to about $5 \%$ of cases of renal cancer and affects men and women equally.

Among the renal cancers this type presents the best prognosis. Histologically it is composed of cells with an abundant, acidophilic cytoplasm, rich in mitochondria, forming vast, solid fields surrounded by connective tissue. A very characteristic morphological feature of chromophobe cell renal carcinoma is a clear area around the nucleus - a formation called a 'halo'[6].

The differentiation towards epithelial cells of the distal tubules of the nephron is also presented in collecting (Bellini) duct carcinoma (CDC) - the most malignant of renal cancers [7].

It is a rapidly growing, aggressive tumor - at the time of diagnosis the presence of metastases is usually encountered. Histologically, it is a two-phase tumor, composed of a mixture of epithelial cells with a significant degree of atypia, embedded in desmoplastically stimulated stroma. Epithelial cells often have features of sarcomatoid dedifferentiation; their grade of malignancy is often classified as 4 on the Fuhrman scale [8].

The next type is medullary renal cell carcinoma - it can be almost exclusively found in children with sickle cell trait. It is very malignant with very high mortality rate.
Medullary cancer cells show rhabdoid differentiation, containing large pleomorphic nuclei with distinct nucleoli, eosinophilic cytoplasm and form irregular nest or trabecular and papillary systems. There are often hemorrhages, areas of the so-called "geographical" necrosis and lymphocytes. The mitoses are frequent $[9,10]$.

In 2012, five more entities of malignant renal neoplasms were proposed during the Consensus Conference ISUP in Vancouver.

Tubulocystic renal cell carcinoma - a rarely occurring tumor, located mainly in the renal cortex, clearly demarcated, with a characteristic macroscopic appearance, resembling a sponge on the cross-section.

Microscopically built of numerous cystic and tubular structures covered with atypical epithelial cells with abundant eosinophilic cytoplasm. This kind of cancer most often shows low malignancy, and the treatment of choice can often be partial nephrectomy [11].

Acquired Cystic Disease -associated RCC is a cancer that occurs in the course of end-stage kidneys, mostly with acquired cystic disease. Usually, found accidentally, it occurs as a tumor or thickening of the cyst wall in the kidney. On the surface of the cross section it is of-ten yellowish-gray, with the presence of calcifications. They are characterized by various his-tological structures - many architectural patterns, including: acinar, tubular, solid-alveolar, microcystic and macrocystic may occur. Most cells have abundant, eosinophilic cytoplasm and large, marked nuclei with the presence of nucleoli. Oxalate crystals are typically present. In low-differentiated cases, sarcomatoid or rhabdoid dedifferentiation may occur $[12,13]$.

Clear cell (tubulo) papillary renal cell carcinoma is a separate group of kidney cancers, distinguished by an immunohistochemical profile.

In the past the features of morphological structure of this tumor suggested different classification - it was once classified as clear cell carcinoma or so-called angiomyoadenomato-sus tumor. However, the immunohistochemical profile, including the positive staining for CK7, CA-IX, high-molecular weight cytokeratin, with the negative staining for CD10 and AMACR, indicates a separate nosological unit [14].

Macroscopically, this is a well-delineated and usually encapsulated tumor, yellowish on the cross-section completely solid to predominantly cystic. Microscopically built from tubules, papillae, cysts or solid areas, with a significant amount of fibrous stroma. The malignancy of the tumor is negligible. MiT Family Translocation RCC is a group of very rare cancers in which we deal with TFE3, TFEB, TFEC and MiTF translocations.

There is a group of heterogeneous tumors in which further studies are necessary to es-tablish final diagnostic criteria. In about 30 cases described in this group, only three have de-veloped into metastasizing forms and are life-threatening for patients $[15,16]$.

\section{Hereditary Leiomyomatosis RCC Syndrome-associated RCC}

This group of renal neoplasms was already mentioned in the 2004 WHO classification, although it was not described as a new entity. Hereditary leiomyomatosis RCC syndrome is autosomal dominant, associated with germline mutation at chromosome $1 \mathrm{q} 42$. 
This is the highly malignant neoplasm with aggressive behavior, patients usually presented with metastases and dissemination, they survival time is short. However recognition of this cancer is very important, as the proper diagnosis gives possibility for appropriate treatment for patient and follow-up of family members $[17,18]$.

\section{Methods of histopathological diagnosis of renal neoplasms}

Concerning the heterogeneity of the structure and histochemical profiles, the final di-agnosis of renal neoplasms has to be based on morphological, immunohistochemical and molecular analysis.

\section{Immunohistochemical stainings}

Ambiguous cases, where the consensus of diagnosis cannot be achieved based on the histopathological image only, additional diagnostic methods are required.

In such cases we usually perform immunohistochemical staining using an antibody panel. Among the most commonly used there are RCC marker, CD 10, Vimentin andCKAE1AE3. This panel of antibodies is often used in the diagnosis of clear cell carcino-ma, in which the co-incidence of Vimentin + and cytokeratin AE1AE3 staining, along with no $\mathrm{CK} 7$ reaction is characteristic. In addition, antibodies such as CK8, CK18, CK19, MUC1, MUC3 are also used in the diagnosis of clear cell carcinoma, a strong reaction is also seen after PAX2 and CA-IX staining [19].

AMACR-Alpha Methyl Acyl Coenzyme A Racemase mitochondrial and peroxiso-mal enzyme involved in betaoxidation of branched fatty acids, currently widely used in the diagnosis of prostate cancer, is also applicable in the differentiation of papillary type I kidney cancer, where the CK7 reaction is most often pronounced strongly, while the positive reaction for $\mathrm{CK} 20$ and e-cadherin $(+)$ is characteristic for type II papillary renal cell carcinoma [20].

Recent genetic studies have revealed prognostic potential of several biomarkers, such as EGFR, p53, VEGF antibodies.

$\mathrm{Ki}-67$ (marker of cell proliferation) is of great importance in the differentiation of ox-yphilic tumors (like oncocytoma).

The research on new antibodies is continuing, more and more attention is given to anti-bodies against antiapoptotic factors, including survivin.

\section{REFERENCES}

1. IACR Polish National Oncology Register.

2. Srigley JR, Delahunt B, Eble JN, et al. More. The International Society of Urological Pa-thology (ISUP) Vancouver Classification of Renal Neoplasia. Am J Surg Pathol. 2013;37(10):1469-89.

3. Rekha PR, Rajendiran S, Rao SI, et al. Histological reclassification, histochemical characterization and c-kit immunoexpression in renal cell carcinoma. Indian J Urol. 2008;343-47.

4. Delahunt B, Eble JN. History of the development of the classification of renal cell neoplasia. Clin Lab Med. 2005;25:231-46.

5. Allory Y, Ouazana D, Boucher E, et al. Papillary renal cell carcinoma. Prognostic value of morphological subtypes in clinicopathologic study of 43 cases. Virchows Arch. 200 3;442:336-42.

6. Eble JN, Togashi K, Pisani P, et al. Renal cell carcinoma. In: J. N. Eble, G. Sauter, J. I. Epstein, I. A. Sesterhenn (ed). Pathology and genetics of human tumours of the urinary and male genital organs. Lyon: IARC Press; 2004. p. 12-4.

7. Polascic TJ, Bostwick DG, Cairns P. Molecular genetics and histopathologic features of adult distal nephron tumors. Urology. 2002;60:941-6.

8. Srigley JR, Eble JN. Collecting duct carcinoma of kidney. Semin Diagn Pathol. 1998;15:54-67.

9. Swartz MA, Karth J, Schneider DT, et al. Renal medullary carcinoma: clinical, pathologic, immunohistochemical, and genetic analysis with pathogenetic implications. Urology. 2002;60:1083-9.

10. Beuselinck B, Lerut E, Wolter P, et al. Sarcomatoid dedifferentiation in metastatic clear cell renal cell carcinoma and outcome on treatment with anti-vascular endothelial growth factor receptor tyrosine kinase inhibitors: a retrospective analysis. Clin Genitourin Canc. 2014;12(5):e205-e214.

11. Moses KA, DeCaro JJ,Osunkoya AO, et al. Tubulocystic carcinoma of the kidney: a case report of natural history and long term follow-up. Scientific World J. 2010;10:586-9.

12. Sule N, Yakupoglu U, Shen SS, et al. Calcium oxalate deposition in renal cell carcinoma associated with acquired cystic kidney disease-associated carcinoma. Am J Surg Pathol. 2012;20:19-28.

13. Kuroda N, Tamura M, Hamaguchi N, et al. Acquired cystic disease-associated renal cell carcinoma with sarcomatoid change and rhabdoid features. Ann Diagn Pathol. 2011;15:462-6.

14. Gobbo S, Eble JN, Grignon DJ, et al. Clear cell papillary renal cell carcinoma: a distinct histopathologic and molecular genetic entity. Am J Surg Pathol. 2008;32:1239-45.

15. Argani P, Lal P, Hutchinson B, et al. Aberrant nuclear immunoreactivity for TFE3 in neoplasms withTFE3 gene fusions: A sensitive and specific immunohistochemical assay. Am J Surg Pathol. 2003;27:750-61.

16. Komai Y, Fujiwara M, Fujii Y, et al. Adult Xp11 translocation renal cel carcinoma diagnosed by cytogenetics and immunohistochemistry. Clin Cancer Res. 2009;15:1170-6.

17. Smith N, Illei P, Gonzales N, et al. Renal cell carcinoma (RCC): report of six new genetically-confirmed cases and expanded Immunohistochemical (IHC) profile. Mod Pathol. 2013;26 (S2):250A

18. Hewett-Emmett D, Tashian RE. Functional diversity, conservation, and convergence in the evolution of the alpha-, beta-, and gamma-carbonic anhydrase gene families. Mol Phylogenet Evol. 1996;5(1):50-77.

19. Immunohistology of prostate, bladder, testis and kidney. In: D. Dabbs (ed). Diagnostic immunohistochemistry. Philadelphia: Churchill Livingstone; 2006.

20. Zhou M, Roma A, Magi-Galluzzi C. The usefulness of immunohistochemical markers in the differential diagnosis of renal cell neoplasms. Clin Lab Med. 2005;25:247-57.

\section{Corresponding author}

Maria Pyda-Karwicka

School of Medicine Jan Kochanowski University, Kielce

5 S. Zeromskiego St., 25-001 Kielce

E-mail: marysiak@poczta.onet.pl 